\title{
Some results for sums of products of Chebyshev and Legendre polynomials
}

\section{Yuan $\mathrm{He}^{1 *}$ (D)}

\section{"Correspondence:}

hyyhe@aliyun.com

'School of Mathematics and

Information Science, Neijiang

Normal University, Neijiang, People's

Republic of China

\begin{abstract}
In this paper, we perform a further investigation of the Gegenbauer polynomials, the Chebyshev polynomials of the first and second kinds and the Legendre polynomials. By making use of some analytic and combinatorial methods, we establish some new expressions for sums of products of arbitrary numbers of Chebyshev polynomials of the first and second kinds and Legendre polynomials.
\end{abstract}

Keywords: Gegenbauer polynomials; Chebyshev polynomials; Legendre polynomials; Combinatorial identities

\section{Introduction}

Gegenbauer [5] in 1874 introduced and studied the Gegenbauer polynomials, which are usually defined for a real number $\lambda>-1 / 2$ with $\lambda \neq 0$ by the generating function

$$
\frac{1}{\left(1-2 x t+t^{2}\right)^{\lambda}}=\sum_{n=0}^{\infty} C_{n}^{\lambda}(x) t^{n}
$$

It is easily seen from (1.1) that the Gegenbauer polynomials satisfy the recurrence relationship

$$
n C_{n}^{\lambda}(x)=2 x(\lambda+n-1) C_{n-1}^{\lambda}(x)-(2 \lambda+n-2) C_{n-2}^{\lambda}(x) \quad(n \geq 2)
$$

with initial values $C_{0}^{\lambda}(x)=1$ and $C_{1}^{\lambda}(x)=2 \lambda x$. In particular, one can derive from Eq. (1.2) the first few expressions for the Gegenbauer polynomials (see, e.g., [6, pp. 990-991]). More precisely, these polynomials can be expressed as (see, e.g., [6, p. 991])

$$
C_{n}^{\lambda}(x)=\frac{\Gamma(n+2 \lambda)}{\Gamma(n+1) \Gamma(2 \lambda)}{ }_{2} F_{1}\left(-n, n+2 \lambda, \lambda+\frac{1}{2} ; \frac{1-x}{2}\right),
$$

where $\Gamma(\cdot)$ is the Gamma function and ${ }_{2} F_{1}(a, b, c ; x)$ is the Gauss hypergeometric function given for complex numbers $a, b, c$ with $c \neq 0,-1,-2, \ldots$ by the infinite series (see, e.g., [1])

$$
{ }_{2} F_{1}(a, b, c ; z)=\sum_{n=0}^{\infty} \frac{a^{(n)} b^{(n)}}{c^{(n)}} \frac{z^{n}}{n !} .
$$

(c) The Author(s) 2019. This article is distributed under the terms of the Creative Commons Attribution 4.0 International License (http://creativecommons.org/licenses/by/4.0/), which permits unrestricted use, distribution, and reproduction in any medium, provided you give appropriate credit to the original author(s) and the source, provide a link to the Creative Commons license, and indicate if changes were made. 
Here $\alpha^{(n)}$ appearing in the above series is the rising factorial given for complex number $\alpha$ and non-negative integer $n$ by $\alpha^{(0)}=1$ and $\alpha^{(n)}=\alpha(\alpha+1) \cdots(\alpha+n-1)$ for positive integer $n$. It is worth noticing that Eq. (1.3) defines the generalized functions $C_{n}^{\lambda}(x)$, where the subscript $n$ is an arbitrary number.

It is also well known that the Gegenbauer polynomials and the Jacobi polynomials, can be connected to the Legendre polynomials and the Chebyshev polynomials. For example, the case $\lambda=1 / 2$ and $\lambda=1$ in (1.1) gives the Legendre polynomials $P_{n}(x)$ and the Chebyshev polynomials of the second kind $U_{n}(x)$, respectively. The Chebyshev polynomials of the first kind $T_{n}(x)$ can be expressed by the Gegenbauer polynomials, as follows (see, e.g., $[1,3]$ ):

$$
\epsilon_{n} T_{n}(x)=\lim _{\lambda \rightarrow 0} \frac{n+\lambda}{\lambda} C_{n}^{\lambda}(x) \quad(n \geq 0),
$$

where $\epsilon_{n}:=2-\delta_{n, 0}$ is the Neumann factor and the Chebyshev polynomials of the first kind are defined by the generating function

$$
\frac{1-x t}{1-2 x t+t^{2}}=\sum_{n=0}^{\infty} T_{n}(x) t^{n}
$$

In recent years, some authors have taken a lively interest in dealing with the expressions for sums of products of arbitrary number of the Chebyshev polynomials and the Legendre polynomials. For example, Zhang [21] used the generating function methods to study the calculating problems of sums of products of arbitrary numbers of Chebyshev polynomials of the first and second kinds and found that, for positive integer $m$ and non-negative integer $n$,

$$
\begin{aligned}
& \sum_{\substack{k_{1}+\cdots+k_{m}=n+m \\
k_{1}, \ldots, k_{m} \geq 0}} T_{k_{1}}(x) \cdots T_{k_{m}}(x) \\
& =\frac{1}{2^{m-1}(m-1) !} \sum_{k=0}^{m}\left(\begin{array}{l}
m \\
k
\end{array}\right)(-x)^{k} U_{n+2 m-1-k}^{(m-1)}(x)
\end{aligned}
$$

and

$$
\sum_{\substack{k_{1}+\cdots+k_{m}=n \\ k_{1}, \ldots, k_{m} \geq 0}} U_{k_{1}}(x) \cdots U_{k_{m}}(x)=\frac{1}{2^{m-1}(m-1) !} U_{n+m-1}^{(m-1)}(x),
$$

where $U_{n}^{(k)}(x)$ denotes the $k$ th derivative of $U_{n}(x)$ with respect to $x$. Kim et al. [11, 14] developed new methods to derive the Fourier expansions for sums of products of arbitrary number of the Chebyshev polynomials of the first and second kinds, by virtue of which they expressed the left sides of (1.6) and (1.7) as linear combinations of the Bernoulli polynomials. More recently, Kim et al. [12] explored the expressions for sums of products of arbitrary number of the Legendre polynomials, and one showed that, for positive integers $m, n$,

$$
\sum_{\substack{k_{1}+\cdots+k_{2 m+1}=n \\ k_{1}, \ldots, k_{2 m+1} \geq 0}} P_{k_{1}}(x) \cdots P_{k_{2 m+1}}(x)
$$




$$
=\frac{1}{(2 m-1) ! !} \sum_{k=0}^{n} \frac{(2 m+2 k-3) ! !}{k !} \triangle_{n+1-k, m+k-1} B_{k}(x),
$$

where $(2 n-1) !$ ! is the double factorial given for non-negative integer $n$ by $(-1) ! !=1$ and $(2 n-1) ! !=(2 n-1)(2 n-3) \cdots 3 \cdot 1$ for positive integer $n, \triangle_{n, m}$ is determined for positive integer $n$ and non-negative integer $m$ by

$$
\triangle_{n, m}=\frac{1}{2^{n+m}(2 m-1) ! !} \sum_{j=0}^{\left[\frac{n-1}{2}\right]}(-1)^{j}\left(\begin{array}{c}
n+m \\
j
\end{array}\right)\left(\begin{array}{c}
2 n+2 m-2 j \\
n+m
\end{array}\right)(n+m-2 j)_{m}
$$

with $(\alpha)_{n}$ the falling factorial given for complex number $\alpha$ and non-negative integer $n$ by $(\alpha)_{0}=1$ and $(\alpha)_{n}=\alpha(\alpha-1) \cdots(\alpha-n+1)$ for positive integer $n$, and $B_{n}(x)$ is for the $n$th Bernoulli polynomials. Shen and Chen [18] evaluated the left side of (1.8) in terms of a recurrence sequence and the Legendre polynomials. Kim et al. [8] further evaluated the left side of (1.8) in terms of the Gauss hypergeometric function and the Legendre polynomials. Similar results can also be found in [8], where the left side of (1.8) is expressed as linear combinations of Hermite polynomials, generalized Laguerre polynomials, Gegenbauer polynomials and Jacobi polynomials, respectively.

Motivated and inspired by the work of the above authors, we perform a further investigation for the Gegenbauer polynomials, the Chebyshev polynomials of the first and second kinds and the Legendre polynomials in this paper. By making use of some analytic and combinatorial methods, we establish some new expressions for sums of products of arbitrary numbers of Chebyshev polynomials of the first and second kinds and Legendre polynomials.

\section{The statement of results}

We firstly give the following closed formula for the Gegenbauer polynomials.

Theorem 2.1 Let $n$ be a non-negative integer. Then, for a real number $\lambda>-1 / 2$ with $\lambda \neq 0$,

$$
C_{n}^{\lambda}(x)=\sum_{k=0}^{n}(-1)^{n-k}\left(\begin{array}{c}
\lambda+k-1 \\
k
\end{array}\right)\left(\begin{array}{c}
k \\
n-k
\end{array}\right)(2 x)^{2 k-n},
$$

where $\left(\begin{array}{l}\alpha \\ k\end{array}\right)$ is the general binomial coefficients given for complex number $\alpha$ and non-negative integer $k$ by

$$
\left(\begin{array}{l}
\alpha \\
0
\end{array}\right)=1, \quad\left(\begin{array}{l}
\alpha \\
k
\end{array}\right)=\frac{\alpha(\alpha-1)(\alpha-2) \cdots(\alpha-k+1)}{k !} \quad(k \geq 1) .
$$

Proof Since for complex number $\alpha$,

$$
(1+t)^{\alpha}=\sum_{n=0}^{\infty}\left(\begin{array}{l}
\alpha \\
n
\end{array}\right) t^{n}
$$

by (1.1) and (2.2) we have

$$
\sum_{n=0}^{\infty} C_{n}^{\lambda}(x) t^{n}=\left(1-2 x t+t^{2}\right)^{-\lambda}=\sum_{j=0}^{\infty}\left(\begin{array}{c}
-\lambda \\
j
\end{array}\right)\left(-2 x t+t^{2}\right)^{j}
$$


Observe that

$$
\left(\begin{array}{c}
-\lambda \\
j
\end{array}\right)=(-1)^{j}\left(\begin{array}{c}
\lambda+j-1 \\
j
\end{array}\right) \quad(j \geq 0)
$$

and the binomial theorem means that, for non-negative integer $j$,

$$
\left(2 x t-t^{2}\right)^{j}=t^{j} \sum_{i=0}^{j}\left(\begin{array}{l}
j \\
i
\end{array}\right)(2 x)^{j-i}(-t)^{i}
$$

It follows from (2.3), (2.4) and (2.5) that

$$
\begin{aligned}
\sum_{n=0}^{\infty} C_{n}^{\lambda}(x) t^{n} & =\sum_{j=0}^{\infty}\left(\begin{array}{c}
\lambda+j-1 \\
j
\end{array}\right) t^{j} \sum_{i=0}^{j}\left(\begin{array}{l}
j \\
i
\end{array}\right)(2 x)^{j-i}(-t)^{i} \\
& =\sum_{j=0}^{\infty}\left(\begin{array}{c}
\lambda+j-1 \\
j
\end{array}\right) t^{j} \sum_{i=j}^{2 j}\left(\begin{array}{c}
j \\
i-j
\end{array}\right)(2 x)^{2 j-i}(-t)^{i-j} \\
& =\sum_{j=0}^{\infty}\left(\begin{array}{c}
\lambda+j-1 \\
j
\end{array}\right) \sum_{i=j}^{2 j}\left(\begin{array}{c}
j \\
i-j
\end{array}\right)(2 x)^{2 j-i}(-1)^{i-j} t^{i} .
\end{aligned}
$$

By changing the order of the summations on the right side of (2.6), we discover

$$
\sum_{n=0}^{\infty} C_{n}^{\lambda}(x) t^{n}=\sum_{i=0}^{\infty} \sum_{j=0}^{i}\left(\begin{array}{c}
\lambda+j-1 \\
j
\end{array}\right)\left(\begin{array}{c}
j \\
i-j
\end{array}\right)(2 x)^{2 j-i}(-1)^{i-j} t^{i}
$$

Thus, comparing the coefficients of $t^{n}$ in (2.7) gives the desired result.

We next provide an alternative proof of Theorem 2.1 .

The second proof of Theorem 2.1 Obviously, we obtain from (1.1)

$$
n ! C_{n}^{\lambda}(x)=\left.\frac{\partial^{n}}{\partial t^{n}}\left(\frac{1}{\left(1-2 x t+t^{2}\right)^{\lambda}}\right)\right|_{t=0} \quad(n \geq 0) .
$$

Let $F(u)=\frac{1}{u^{\lambda}}$ and $G(t)=1-2 x t+t^{2}$ with $u=G(t)$. The famous Faà di Bruno formula implies that, for positive integer $n$, (see, e.g., [4, pp. 137-139]

$$
F^{(n)}(G(t))=\sum_{k=1}^{n} F^{(k)}(u) B_{n, k}\left(G^{(1)}(t), G^{(2)}(t), \ldots, G^{(n-k+1)}(t)\right),
$$

where $f^{(n)}(x)$ denotes the $n$th derivative for the function $f(x)$ with respect to $x$, and $B_{n, k}\left(x_{1}, x_{2}, \ldots, x_{n-k+1}\right)$ is for the partial Bell polynomials given for positive integers $n, k$ with $n \geq k$ by (see, e.g., [4, pp. 134-135])

$$
B_{n, k}\left(x_{1}, x_{2}, \ldots, x_{n-k+1}\right)=\sum \frac{n !}{j_{1} ! j_{2} ! j_{3} ! \cdots}\left(\frac{x_{1}}{1 !}\right)^{j_{1}}\left(\frac{x_{2}}{2 !}\right)^{j_{2}}\left(\frac{x_{3}}{3 !}\right)^{j_{3}} \ldots
$$


Note that the above summation takes place over non-negative integers $j_{1}, j_{2}, j_{3}, \ldots$ such that

$$
j_{1}+2 j_{2}+3 j_{3}+\cdots=n \text { and } j_{1}+j_{2}+j_{3}+\cdots=k
$$

Observe that, for positive integer $k$,

$$
\begin{aligned}
F^{(k)}(u) & =(-\lambda)(-\lambda-1) \cdots(-\lambda-k+1) u^{-\lambda-k} \\
& =(-1)^{k} k !\left(\begin{array}{c}
\lambda+k-1 \\
k
\end{array}\right) \frac{1}{\left(1-2 x t+t^{2}\right)^{\lambda+k}} .
\end{aligned}
$$

It follows from (2.9) and (2.10) that, for positive integer $n$,

$$
\begin{aligned}
& \left.\frac{\partial^{n}}{\partial t^{n}}\left(\frac{1}{\left(1-2 x t+t^{2}\right)^{\lambda}}\right)\right|_{t=0} \\
& \quad=\sum_{k=1}^{n}(-1)^{k} k !\left(\begin{array}{c}
\lambda+k-1 \\
k
\end{array}\right) B_{n, k}(-2 x, 2,0, \ldots, 0) .
\end{aligned}
$$

It is easily seen from the definition of the partial Bell polynomials that

$$
B_{n, k}(-2 x, 2,0, \ldots, 0)=\frac{n !}{(2 k-n) !(n-k) !}(-2 x)^{2 k-n} .
$$

Thus, by applying (2.12) to (2.11), in the light of (2.8) and $C_{0}^{\lambda}(x)=1$, we complete the proof of Theorem 2.1.

There follows an expression for the Gegenbauer polynomials in terms of the Stirling numbers of the first kind.

Corollary 2.2 Let $n$ be a non-negative integer. Then, for a real number $\lambda>-1 / 2$ with $\lambda \neq 0$,

$$
C_{n}^{\lambda}(x)=(-1)^{n} \sum_{k=0}^{\left[\frac{n}{2}\right]} \frac{(2 x)^{n-2 k}}{k !(n-2 k) !} \sum_{j=0}^{n-k} s(n-k, j)(-\lambda)^{j}
$$

where $s(n, k)$ the Stirling numbers of the first kind given for non-negative integers $n, k$ with $0 \leq k \leq n$ by the generating function (see, e.g., [4])

$$
\frac{(\ln (1+t))^{k}}{k !}=\sum_{n=k}^{\infty} s(n, k) \frac{t^{n}}{n !} .
$$

Proof It is clear from Theorem 2.1 that

$$
\begin{aligned}
C_{n}^{\lambda}(x) & =\sum_{k=0}^{n}(-1)^{k}\left(\begin{array}{c}
\lambda+n-k-1 \\
n-k
\end{array}\right)\left(\begin{array}{c}
n-k \\
k
\end{array}\right)(2 x)^{n-2 k} \\
& =\sum_{k=0}^{\left[\frac{n}{2}\right]}(-1)^{k} \frac{(2 x)^{n-2 k}}{k !(n-2 k) !}(\lambda+n-k-1) \cdots(\lambda+1) \lambda .
\end{aligned}
$$


Notice that the Stirling numbers of the first kind can be characterized by the identity (see, e.g., [4, p. 213])

$$
x(x-1) \cdots(x-n+1)=\sum_{j=0}^{n} s(n, j) x^{j} \quad(n \geq 0),
$$

where the left side of the above identity is equal to 1 when $n=0$. By taking $x=-\lambda$ in (2.15), we have

$$
(-1)^{n} \lambda(\lambda+1) \cdots(\lambda+n-1)=\sum_{j=0}^{n} s(n, j)(-\lambda)^{j} \quad(n \geq 0) .
$$

Thus, by replacing $n$ by $n-k$ in (2.16), with the help of (2.14), we get the desired result immediately.

We are now in the position to evaluate the sums of products of arbitrary number of Chebyshev polynomials of the first and second kinds and Legendre polynomials in terms of the Stirling numbers of the first kind.

Corollary 2.3 Let $m, n$ be non-negative integers with $m \geq 1$. Then

$$
\begin{aligned}
& \sum_{\substack{k_{1}+\cdots+k_{m}=n \\
k_{1}, \ldots, k_{m} \geq 0}} P_{k_{1}}(x) \cdots P_{k_{m}}(x) \\
& =(-1)^{n} \sum_{k=0}^{\left[\frac{n}{2}\right]} \frac{(2 x)^{n-2 k}}{k !(n-2 k) !} \sum_{j=0}^{n-k} \frac{s(n-k, j)(-m)^{j}}{2^{j}}
\end{aligned}
$$

and

$$
\begin{aligned}
& \sum_{\substack{k_{1}+\cdots+k_{m}=n \\
k_{1}, \ldots, k_{m} \geq 0}} U_{k_{1}}(x) \cdots U_{k_{m}}(x) \\
& =(-1)^{n} \sum_{k=0}^{\left[\frac{n}{2}\right]} \frac{(2 x)^{n-2 k}}{k !(n-2 k) !} \sum_{j=0}^{n-k} s(n-k, j)(-m)^{j} .
\end{aligned}
$$

Proof We know from the definitions of the Legendre polynomials and the Chebyshev polynomials of the second kind that

$$
\left(\sum_{n=0}^{\infty} P_{n}(x) t^{n}\right)^{m}=\frac{1}{\left(1-2 x t+t^{2}\right)^{\frac{m}{2}}}=\sum_{n=0}^{\infty} C_{n}^{\frac{m}{2}}(x) t^{n}
$$

and

$$
\left(\sum_{n=0}^{\infty} U_{n}(x) t^{n}\right)^{m}=\frac{1}{\left(1-2 x t+t^{2}\right)^{m}}=\sum_{n=0}^{\infty} C_{n}^{m}(x) t^{n} .
$$


If we apply the Cauchy product and compare the coefficients of $t^{n}$ in the above two identities, we obtain

$$
\sum_{\substack{k_{1}+\cdots+k_{m}=n \\ k_{1}, \ldots, k_{m} \geq 0}} P_{k_{1}}(x) \cdots P_{k_{m}}(x)=C_{n}^{\frac{m}{2}}(x)
$$

and

$$
\sum_{\substack{k_{1}+\cdots+k_{m}=n \\ k_{1}, \ldots, k_{m} \geq 0}} U_{k_{1}}(x) \cdots U_{k_{m}}(x)=C_{n}^{m}(x)
$$

Hence, by taking $\lambda=m / 2$ and $m$ in Corollary 2.2, in view of (2.19) and (2.20), we complete the proof of Corollary 2.3.

Corollary 2.4 Let $m, n$ be positive integers with $n \geq m$. Then

$$
\begin{aligned}
& \sum_{\substack{k_{1}+\cdots+k_{m}=n \\
k_{1}, \ldots, k_{m} \geq 0}} T_{k_{1}}(x) \cdots T_{k_{m}}(x) \\
& =(-1)^{n} \sum_{k=0}^{m}\left(\begin{array}{c}
m \\
k
\end{array}\right) x^{k} \sum_{j=0}^{\left[\frac{n-k}{2}\right]} \frac{(2 x)^{n-k-2 j}}{j !(n-k-2 j) !} \\
& \quad \times \sum_{l=0}^{n-k-j} s(n-k-j, l)(-m)^{l} .
\end{aligned}
$$

Proof It is clear from (1.5) and the binomial theorem that

$$
\begin{aligned}
\left(\sum_{n=0}^{\infty} T_{n}(x) t^{n}\right)^{m} & =\frac{(1-x t)^{m}}{\left(1-2 x t+t^{2}\right)^{m}} \\
& =\sum_{k=0}^{m}\left(\begin{array}{c}
m \\
k
\end{array}\right)(-x)^{k} \sum_{n=0}^{\infty} C_{n}^{m}(x) t^{n+k} .
\end{aligned}
$$

Comparing the coefficients of $t^{n}$ in the above identity gives

$$
\sum_{\substack{k_{1}+\cdots+k_{m}=n \\
k_{1}, \ldots, k_{m} \geq 0}} T_{k_{1}}(x) \cdots T_{k_{m}}(x)=\sum_{k=0}^{m}\left(\begin{array}{c}
m \\
k
\end{array}\right)(-x)^{k} C_{n-k}^{m}(x) .
$$

Thus, by applying Corollary 2.2 to (2.23), we get the desired result.

For some related results about sums of products of the Chebyshev polynomials, see [2, 7 , 9, 10, 13, 15-17, 19, 20]. Based on the work of Shen and Chen [18], and Kim et al. [8], who evaluated the left side of (1.8) in terms of the Legendre polynomials, we next give other expressions for the sums of products of arbitrary numbers of Chebyshev polynomials of the first and second kinds and Legendre polynomials. To do this, we present the following results. 
Theorem 2.5 Let $n$ be a positive integer. Then, for a real number $m>-1$ with $m \neq 0$,

$$
C_{n}^{\frac{m}{2}}(x)=\sum_{k=1}^{n}\left(\frac{k(m+1)}{n}-1\right) P_{k}(x) C_{n-k}^{\frac{m}{2}}(x),
$$

and, for a real number $\lambda>-1 / 2$ with $\lambda \neq 0$,

$$
C_{n}^{\lambda}(x)=\sum_{k=1}^{n}\left(\frac{k(\lambda+1)}{n}-1\right) U_{k}(x) C_{n-k}^{\lambda}(x) .
$$

Proof Let $f(t)$ be a function of $t$. Then, for positive integer $n$,

$$
\begin{aligned}
\frac{\partial^{n}}{\partial t^{n}}\left(\frac{1}{(f(t))^{\lambda+1}}\right) & =\frac{\partial^{n-1}}{\partial t^{n-1}}\left\{\frac{\partial}{\partial t}\left(\frac{1}{(f(t))^{\lambda+1}}\right)\right\} \\
& =\frac{\partial^{n-1}}{\partial t^{n-1}}\left\{\frac{\lambda+1}{(f(t))^{\lambda}} \frac{\partial}{\partial t}\left(\frac{1}{f(t)}\right)\right\},
\end{aligned}
$$

which together with the Leibniz rule yields

$$
\begin{aligned}
& \frac{\partial^{n}}{\partial t^{n}}\left(\frac{1}{(f(t))^{\lambda+1}}\right) \\
& \quad=(\lambda+1) \sum_{k=0}^{n-1}\left(\begin{array}{c}
n-1 \\
k
\end{array}\right) \frac{\partial^{k+1}}{\partial t^{k+1}}\left(\frac{1}{f(t)}\right) \frac{\partial^{n-1-k}}{\partial t^{n-1-k}}\left(\frac{1}{(f(t))^{\lambda}}\right) \\
& \quad=(\lambda+1) \sum_{k=1}^{n}\left(\begin{array}{c}
n-1 \\
k-1
\end{array}\right) \frac{\partial^{k}}{\partial t^{k}}\left(\frac{1}{f(t)}\right) \frac{\partial^{n-k}}{\partial t^{n-k}}\left(\frac{1}{(f(t))^{\lambda}}\right)
\end{aligned}
$$

On the other hand, for positive integer $n$,

$$
\frac{\partial^{n}}{\partial t^{n}}\left(\frac{1}{(f(t))^{\lambda+1}}\right)=\sum_{k=0}^{n}\left(\begin{array}{l}
n \\
k
\end{array}\right) \frac{\partial^{k}}{\partial t^{k}}\left(\frac{1}{f(t)}\right) \frac{\partial^{n-k}}{\partial t^{n-k}}\left(\frac{1}{(f(t))^{\lambda}}\right)
$$

It follows from (2.26) and (2.27) that, for positive integer $n$,

$$
\begin{gathered}
(\lambda+1) \sum_{k=1}^{n}\left(\begin{array}{l}
n-1 \\
k-1
\end{array}\right) \frac{\partial^{k}}{\partial t^{k}}\left(\frac{1}{f(t)}\right) \frac{\partial^{n-k}}{\partial t^{n-k}}\left(\frac{1}{(f(t))^{\lambda}}\right) \\
=\sum_{k=0}^{n}\left(\begin{array}{l}
n \\
k
\end{array}\right) \frac{\partial^{k}}{\partial t^{k}}\left(\frac{1}{f(t)}\right) \frac{\partial^{n-k}}{\partial t^{n-k}}\left(\frac{1}{(f(t))^{\lambda}}\right),
\end{gathered}
$$

which means

$$
\begin{aligned}
\frac{1}{f(t)} & \frac{\partial^{n}}{\partial t^{n}}\left(\frac{1}{(f(t))^{\lambda}}\right) \\
= & (\lambda+1) \sum_{k=1}^{n}\left(\begin{array}{l}
n-1 \\
k-1
\end{array}\right) \frac{\partial^{k}}{\partial t^{k}}\left(\frac{1}{f(t)}\right) \frac{\partial^{n-k}}{\partial t^{n-k}}\left(\frac{1}{(f(t))^{\lambda}}\right) \\
& -\sum_{k=1}^{n}\left(\begin{array}{l}
n \\
k
\end{array}\right) \frac{\partial^{k}}{\partial t^{k}}\left(\frac{1}{f(t)}\right) \frac{\partial^{n-k}}{\partial t^{n-k}}\left(\frac{1}{(f(t))^{\lambda}}\right)
\end{aligned}
$$




$$
=\sum_{k=1}^{n}\left(\begin{array}{l}
n \\
k
\end{array}\right)\left(\frac{k(\lambda+1)}{n}-1\right) \frac{\partial^{k}}{\partial t^{k}}\left(\frac{1}{f(t)}\right) \frac{\partial^{n-k}}{\partial t^{n-k}}\left(\frac{1}{(f(t))^{\lambda}}\right) .
$$

If we take $\lambda=m$ and $f(t)=\sqrt{1-2 x t+t^{2}}$ in (2.28) then, for positive integer $n$,

$$
\begin{aligned}
& \left.\frac{\partial^{n}}{\partial t^{n}}\left(\frac{1}{\left(1-2 x t+t^{2}\right)^{\frac{m}{2}}}\right)\right|_{t=0} \\
& =\left.\sum_{k=1}^{n}\left(\begin{array}{l}
n \\
k
\end{array}\right)\left(\frac{k(m+1)}{n}-1\right) \frac{\partial^{k}}{\partial t^{k}}\left(\frac{1}{\sqrt{1-2 x t+t^{2}}}\right)\right|_{t=0} \\
& \quad \times\left.\frac{\partial^{n-k}}{\partial t^{n-k}}\left(\frac{1}{\left(1-2 x t+t^{2}\right)^{\frac{m}{2}}}\right)\right|_{t=0},
\end{aligned}
$$

and if we take $f(t)=1-2 x t+t^{2}$ in (2.28) then, for positive integer $n$,

$$
\begin{aligned}
\left.\frac{\partial^{n}}{\partial t^{n}}\left(\frac{1}{\left(1-2 x t+t^{2}\right)^{\lambda}}\right)\right|_{t=0}= & \left.\sum_{k=1}^{n}\left(\begin{array}{l}
n \\
k
\end{array}\right)\left(\frac{k(\lambda+1)}{n}-1\right) \frac{\partial^{k}}{\partial t^{k}}\left(\frac{1}{1-2 x t+t^{2}}\right)\right|_{t=0} \\
& \times\left.\frac{\partial^{n-k}}{\partial t^{n-k}}\left(\frac{1}{\left(1-2 x t+t^{2}\right)^{\lambda}}\right)\right|_{t=0} .
\end{aligned}
$$

Thus, applying (2.8) to (2.29) and (2.30) gives (2.24) and (2.25), respectively.

Corollary 2.6 Let $m, n$ be positive integers. Then

$$
\begin{aligned}
& \sum_{\substack{k_{1}+\cdots+k_{m}=n \\
k_{1}, \ldots, k_{m} \geq 0}} P_{k_{1}}(x) \cdots P_{k_{m}}(x) \\
& =\sum_{k=1}^{n}\left(\frac{(m+1)(n+1-k)}{n}-1\right) P_{n+1-k}(x) \\
& \quad \times \sum_{j=0}^{k-1}(-1)^{k-1-j}\left(\begin{array}{c}
\frac{m}{2}+j-1 \\
j
\end{array}\right)\left(\begin{array}{c}
j \\
k-1-j
\end{array}\right)(2 x)^{2 j+1-k}
\end{aligned}
$$

and

$$
\begin{aligned}
& \sum_{\substack{k_{1}+\cdots+k_{m}=n \\
k_{1}, \ldots, k_{m} \geq 0}} U_{k_{1}}(x) \cdots U_{k_{m}}(x) \\
& =\sum_{k=1}^{n}\left(\frac{(m+1)(n+1-k)}{n}-1\right) U_{n+1-k}(x) \\
& \quad \times \sum_{j=0}^{k-1}(-1)^{k-1-j}\left(\begin{array}{c}
m+j-1 \\
j
\end{array}\right)\left(\begin{array}{c}
j \\
k-1-j
\end{array}\right)(2 x)^{2 j+1-k} .
\end{aligned}
$$

Proof By applying Theorem 2.5 to the right sides of (2.19) and (2.20), we discover

$$
\sum_{\substack{k_{1}+\cdots+k_{m}=n \\ k_{1}, \ldots, k_{m} \geq 0}} P_{k_{1}}(x) \cdots P_{k_{m}}(x)=\sum_{k=1}^{n}\left(\frac{k(m+1)}{n}-1\right) P_{k}(x) C_{n-k}^{\frac{m}{2}}(x)
$$


and

$$
\sum_{\substack{k_{1}+\cdots+k_{m}=n \\ k_{1}, \ldots, k_{m} \geq 0}} U_{k_{1}}(x) \cdots U_{k_{m}}(x)=\sum_{k=1}^{n}\left(\frac{k(m+1)}{n}-1\right) U_{k}(x) C_{n-k}^{m}(x)
$$

and it follows from Theorem 2.1 that

$$
\begin{aligned}
& \sum_{\substack{k_{1}+\cdots+k_{m}=n \\
k_{1}, \ldots, k_{m} \geq 0}} P_{k_{1}}(x) \cdots P_{k_{m}}(x) \\
& =\sum_{k=1}^{n}\left(\frac{k(m+1)}{n}-1\right) P_{k}(x) \\
& \quad \times \sum_{j=0}^{n-k}(-1)^{n-k-j}\left(\begin{array}{c}
\frac{m}{2}+j-1 \\
j
\end{array}\right)\left(\begin{array}{c}
j \\
n-k-j
\end{array}\right)(2 x)^{2 j-(n-k)}
\end{aligned}
$$

and

$$
\begin{aligned}
& \sum_{\substack{k_{1}+\cdots+k_{m}=n \\
k_{1}, \ldots, k_{m} \geq 0}} U_{k_{1}}(x) \cdots U_{k_{m}}(x) \\
& =\sum_{k=1}^{n}\left(\frac{k(m+1)}{n}-1\right) U_{k}(x) \\
& \quad \times \sum_{j=0}^{n-k}(-1)^{n-k-j}\left(\begin{array}{c}
m+j-1 \\
j
\end{array}\right)\left(\begin{array}{c}
j \\
n-k-j
\end{array}\right)(2 x)^{2 j-(n-k)} .
\end{aligned}
$$

Thus, by replacing $k$ by $n+1-k$ in the right sides of (2.33) and (2.34), we get the desired results.

Corollary 2.7 Let $m, n$ be positive integers with $n \geq m+1$. Then

$$
\begin{aligned}
& \sum_{\substack{k_{1}+\cdots+k_{m}=n \\
k_{1}, \ldots, k_{m} \geq 0}} T_{k_{1}}(x) \cdots T_{k_{m}}(x) \\
& =\sum_{k=1}^{n}\left(\frac{(m+1)(n+1-k)}{n}-1\right) T_{n+1-k}(x) \sum_{j=0}^{m}\left(\begin{array}{c}
m \\
j
\end{array}\right)(-x)^{j} \\
& \quad \times \sum_{l=0}^{k-1-j}(-1)^{k-1-j-l}\left(\begin{array}{c}
m+l-1 \\
l
\end{array}\right)\left(\begin{array}{c}
l \\
k-1-j-l
\end{array}\right)(2 x)^{2 l+j+1-k}
\end{aligned}
$$

and

$$
\begin{aligned}
& \sum_{\substack{k_{1}+\cdots+k_{m}=n \\
k_{1}, \ldots, k_{m} \geq 0}} T_{k_{1}}(x) \cdots T_{k_{m}}(x) \\
& =\sum_{k=0}^{m}\left(\begin{array}{l}
m \\
k
\end{array}\right)(-x)^{k} \sum_{j=1}^{n-k}\left(\frac{(m+1)(n+1-k-j)}{n-k}-1\right) U_{n+1-k-j}(x)
\end{aligned}
$$




$$
\times \sum_{l=0}^{j-1}(-1)^{j-1-l}\left(\begin{array}{c}
m+l-1 \\
l
\end{array}\right)\left(\begin{array}{c}
l \\
j-1-l
\end{array}\right)(2 x)^{2 l+1-j}
$$

Proof By taking $\lambda=m$ and

$$
f(t)=\frac{1-2 x t+t^{2}}{1-x t}
$$

in (2.28), we obtain

$$
\begin{aligned}
& \left.\frac{\partial^{n}}{\partial t^{n}}\left(\frac{(1-x t)^{m}}{\left(1-2 x t+t^{2}\right)^{m}}\right)\right|_{t=0} \\
& =\left.\sum_{k=1}^{n}\left(\begin{array}{l}
n \\
k
\end{array}\right)\left(\frac{k(m+1)}{n}-1\right) \frac{\partial^{k}}{\partial t^{k}}\left(\frac{1-x t}{1-2 x t+t^{2}}\right)\right|_{t=0} \\
& \quad \times\left.\frac{\partial^{n-k}}{\partial t^{n-k}}\left(\frac{(1-x t)^{m}}{\left(1-2 x t+t^{2}\right)^{m}}\right)\right|_{t=0} .
\end{aligned}
$$

It is obvious from (2.22) and (2.23) that

$$
\begin{aligned}
\left.\frac{\partial^{n}}{\partial t^{n}}\left(\frac{(1-x t)^{m}}{\left(1-2 x t+t^{2}\right)^{m}}\right)\right|_{t=0}=n ! \sum_{\substack{k_{1}+\cdots+k_{m}=n \\
k_{1}, \ldots, k_{m} \geq 0}} T_{k_{1}}(x) \cdots T_{k_{m}}(x) \\
=n ! \sum_{k=0}^{m}\left(\begin{array}{c}
m \\
k
\end{array}\right)(-x)^{k} C_{n-k}^{m}(x) .
\end{aligned}
$$

It follows from (2.37), (2.38) and Theorem 2.1 that

$$
\begin{aligned}
& \sum_{\substack{k_{1}+\cdots+k_{m}=n \\
k_{1}, \ldots, k_{m} \geq 0}} T_{k_{1}}(x) \cdots T_{k_{m}}(x) \\
& =\sum_{k=1}^{n}\left(\frac{k(m+1)}{n}-1\right) T_{k}(x) \sum_{j=0}^{m}\left(\begin{array}{c}
m \\
j
\end{array}\right)(-x)^{j} C_{n-k-j}^{m}(x) \\
& =\sum_{k=1}^{n}\left(\frac{(m+1)(n+1-k)}{n}-1\right) T_{n+1-k}(x) \\
& \times \sum_{j=0}^{m}\left(\begin{array}{c}
m \\
j
\end{array}\right)(-x)^{j} C_{k-1-j}^{m}(x) \\
& =\sum_{k=1}^{n}\left(\frac{(m+1)(n+1-k)}{n}-1\right) T_{n+1-k}(x) \sum_{j=0}^{m}\left(\begin{array}{c}
m \\
j
\end{array}\right)(-x)^{j} \\
& \times \sum_{l=0}^{k-1-j}(-1)^{k-1-j-l}\left(\begin{array}{c}
m+l-1 \\
l
\end{array}\right)\left(\begin{array}{c}
l \\
k-1-j-l
\end{array}\right)(2 x)^{2 l+j+1-k} .
\end{aligned}
$$

Similarly, from (2.25), (2.38) and Theorem 2.1, we have

$$
\sum_{\substack{k_{1}+\cdots+k_{m}=n \\ k_{1}, \ldots, k_{m} \geq 0}} T_{k_{1}}(x) \cdots T_{k_{m}}(x)
$$




$$
\begin{aligned}
= & \sum_{k=0}^{m}\left(\begin{array}{l}
m \\
k
\end{array}\right)(-x)^{k} \sum_{j=1}^{n-k}\left(\frac{j(m+1)}{n-k}-1\right) U_{j}(x) C_{n-k-j}^{m}(x) \\
= & \sum_{k=0}^{m}\left(\begin{array}{c}
m \\
k
\end{array}\right)(-x)^{k} \sum_{j=1}^{n-k}\left(\frac{(m+1)(n+1-k-j)}{n-k}-1\right) U_{n+1-k-j}(x) C_{j-1}^{m}(x) \\
= & \sum_{k=0}^{m}\left(\begin{array}{l}
m \\
k
\end{array}\right)(-x)^{k} \sum_{j=1}^{n-k}\left(\frac{(m+1)(n+1-k-j)}{n-k}-1\right) U_{n+1-k-j}(x) \\
& \times \sum_{l=0}^{j-1}(-1)^{j-1-l}\left(\begin{array}{c}
m+l-1 \\
l
\end{array}\right)\left(\begin{array}{c}
l \\
j-1-l
\end{array}\right)(2 x)^{2 l+1-j} .
\end{aligned}
$$

This completes the proof of Corollary 2.7.

\section{Conclusions}

In this paper, we establish some new expressions for sums of products of arbitrary numbers of Chebyshev polynomials of the first and second kinds and Legendre polynomials by making use of some analytic and combinatorial methods. The methods presented here may be applied to other families of special polynomials, for example, one could consider the Fibonacci and Lucas polynomials and their generalizations instead. In addition, one can derive some analogous expressions for sums of products of arbitrary number of the Catalan numbers, the central Delannoy numbers and the Schröder numbers, having developed the methods showed in this paper.

Acknowledgements

The author expresses his sincere gratitude to the referees for their careful reading and helpful comments.

Funding

This research received no external funding.

Availability of data and materials

Not applicable.

Competing interests

The author declares no conflict of interest.

Authors' contributions

The author obtained the main results and completed all the parts of this manuscript. The author read and approved the final manuscript.

\section{Publisher's Note}

Springer Nature remains neutral with regard to jurisdictional claims in published maps and institutional affiliations.

Received: 26 June 2019 Accepted: 19 August 2019 Published online: 27 August 2019

\section{References}

1. Andrews, G.E., Askey, R., Roy, R.: Special Functions. Cambridge Univ. Press, Cambridge (1999)

2. Cesarano, C.: Identities and generating functions on Chebyshev polynomials. Georgian Math. J. 19, 427-440 (2012)

3. Cohl, H.S., Costas-Santos, R.S., Wakhare, T.V.: On a generalization of the Rogers generating function. J. Math. Anal. Appl. 475, 1019-1043 (2019)

4. Comtet, L.: Advanced Combinatorics, the Art of Finite and Infinite Expansions. Reidel, Dordrecht (1974)

5. Gegenbauer, L.: Über einige bestimmte Integrale. Sitz. Math. Natur. Kl. Akad. Wiss. Wien (Ila) 70, 433-443 (1874)

6. Jeffrey, A., Zwillinger, D.: Table of Integrals, Series and Products, 7th edn. Academic Press, San Diego (2007)

7. Kim, T., Dolgy, D.V., Kim, D.S.: Representing sums of finite products of Chebyshev polynomials of the second kind and Fibonacci polynomials in terms of Chebyshev polynomials. Adv. Stud. Contemp. Math. 28, 321-335 (2018)

8. Kim, T., Hwang, K.-W., Kim, D.S., Dolgy, D.V.: Connection problem for sums of finite products of Legendre and Laguerre polynomials. Symmetry 11, Article ID 317 (2019) 
9. Kim, T., Kim, D.S., Dolgy, D.V., Kim, D.: Representation by several orthogonal polynomials for sums of finite products of Chebyshev polynomials of the first, third and fourth kinds. Adv. Differ. Equ. 2019, Article ID 110 (2019)

10. Kim, T., Kim, D.S., Dolgy, D.V., Kwon, J.: Sums of finite products of Chebyshev polynomials of the third and fourth kinds. Adv. Differ. Equ. 2018, Article ID 283 (2018)

11. Kim, T., Kim, D.S., Dolgy, D.V., Park, J.-W.: Sums of finite products of Chebyshev polynomials of the second kind and of Fibonacci polynomials. J. Inequal. Appl. 2018, Article ID 148 (2018)

12. Kim, T., Kim, D.S., Dolgy, D.V., Park, J.-W.: Sums of finite products of Legendre and Laguerre polynomials. Adv. Differ. Equ. 2018, Article ID 277 (2018)

13. Kim, T., Kim, D.S., Jang, L.-C., Dolgy, D.V.: Representation by Chebyshev polynomials for sums of finite products of Chebyshev polynomials. Symmetry 10, Article ID 742 (2018)

14. Kim, T., Kim, D.S., Jang, L.-C., Jang, G.-W.: Fourier series for functions related to Chebyshev polynomials of the first kind and Lucas polynomials. Mathematics 6, Article ID 276 (2018)

15. Kim, T., Kim, D.S., Kwon, J., Jang, G.-W.: Sums of finite products of Legendre and Laguerre polynomials by Chebyshev polynomials. Adv. Stud. Contemp. Math. 28, 551-565 (2018)

16. Lee, C.-L., Wong, K.B.: On Chebyshev's polynomials and certain combinatorial identities. Bull. Malays. Math. Sci. Soc. $34,279-286(2011)$

17. Li, X.: Some identities involving Chebyshev polynomials. Math. Probl. Eng. 2015, Article ID 950695 (2015)

18. Shen, S., Chen, L.: Some types of identities involving the Legendre polynomials. Mathematics 7, Article ID 114 (2019)

19. Wang, S.: Some new identities of Chebyshev polynomials and their applications. Adv. Differ. Equ. 2015, Article ID 355 (2015)

20. Wang, T., Zhang, H.: Some identities involving the derivative of the first kind Chebyshev polynomials. Math. Probl. Eng. 2015, Article ID 146313 (2015)

21. Zhang, W.: Some identities involving the Fibonacci numbers and Lucas numbers. Fibonacci Q. 42, 149-154 (2004)

\section{Submit your manuscript to a SpringerOpen ${ }^{\circ}$ journal and benefit from:}

- Convenient online submission

- Rigorous peer review

Open access: articles freely available online

- High visibility within the field

- Retaining the copyright to your article

Submit your next manuscript at $\gg$ springeropen.com 